\title{
Novel fibrillin-1 mutation with variable presentation as a thoracic aortic aneurysm and intramural hematoma
}

\author{
Anand Brahmandam, MBBS, ${ }^{\mathrm{a}}$ and Prashanth Vallabhajosyula, MD, MS, ${ }^{\mathrm{b}}$ New Haven, Conn
}

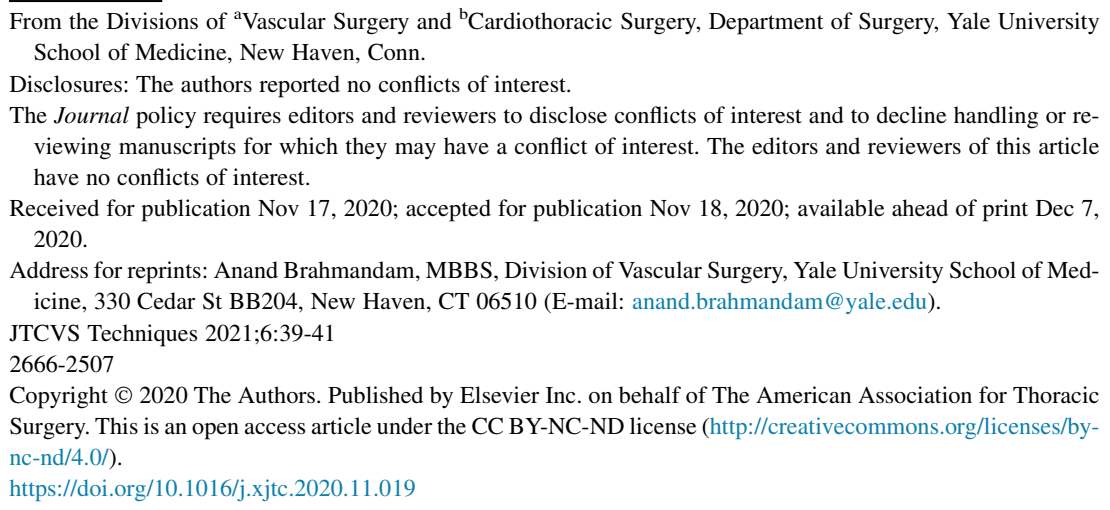

\section{CASE 1}

A 36-year-old male nonsmoker with history of hypertension, hyperlipidemia, morbid obesity (body mass index $=$ $38.2 \mathrm{~kg} / \mathrm{m}^{2}$ ), gout, and asthma presented with a 10-day history of cough and class II heart failure. A chest radiograph obtained by his primary care physician was notable for aortic dilation. Computed tomography (CT) scan of the chest revealed a $6.9 \mathrm{~cm} \times 7.3 \mathrm{~cm}$ (Figure 1, $A$ and $B$ ) aortic root aneurysm. Echocardiography demonstrated a tricuspid aortic valve with severe aortic insufficiency and ejection fraction of $40 \%$. Coincidentally, on the same day the patient's mother (case 2) was admitted with an acute type B intramural hematoma (IMH). There were no overt features of connective tissue disorders. Both patients were genetically tested. Extended gene analysis was notable for an autosomal-dominant missense variant of the fibrillin-1 gene with a predicted deleterious effect on protein function (FBN1: c.T556C: p.C186R). The patient underwent a valve-sparing root reimplantation with transverse hemiarch reconstruction under deep hypothermic circulatory arrest with retrograde cerebral perfusion (proximal: $32 \mathrm{~mm}$; distal: 26 mm; Gelweave Valsalva graft; Terumo Aortic, Sunrise, Fla). His postoperative course was uneventful, and postoperative echocardiography at 1-month showed trace aortic insufficiency with improvement in ejection fraction $(55 \%)$.

\section{CASE 2}

A 69-year-old female nonsmoker with a history of hyperlipidemia presented to the hospital with acute onset of substernal chest and back pain. Her heart rate on presentation was 75 beats per minute, with a blood pressure of 148/

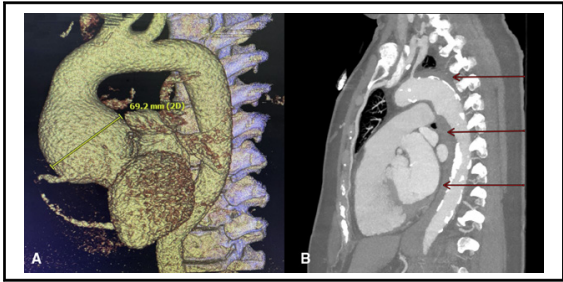

A novel fibrillin-1 gene mutation causing $A$, aortic aneurysm and $B$, intramural hematoma.

\section{CENTRAL MESSAGE \\ This report describes a rare variant mutation of the fibrillin-1 gene and its phenotypic effects on aortic wall homeostasis in 2 related family members.}

See Commentaries on pages 42 and 44 .

$78 \mathrm{~mm} \mathrm{Hg}$, which was symmetric in both upper extremities. CT scan revealed a type B IMH (maximal size $6 \mathrm{~mm}$ ) originating distal to the origin of the left subclavian artery extending to the renal arteries. There was no clinical or radiographic evidence of malperfusion. The patient was admitted for anti-impulse control with esmolol and nicardipine drips. By hospital day 3, she was pain free and transferred to the floor. A repeat CT revealed IMH expansion (maximal size $14 \mathrm{~mm}$ ) and distal extension past the aortoiliac bifurcation with a new area of contrast entry into the IMH. Based on this, she was urgently taken to the operating room for a thoracic endovascular aortic repair. Ultrasoundguided access of bilateral femoral arteries was obtained and an aortogram confirmed the IMH, with its entry site distal to the left subclavian artery, which was widely patent without stenosis. Thoracic endovascular aortic repair was performed using the Relay Plus stent-graft (Terumo Aortic). First, the distal $28 \times 24 \times 150$-mm component was deployed just above the celiac axis, followed by $36 \times 36 \times 150$-mm proximal stent-graft. Completion angiography showed coverage of the IMH entry tear, no evidence of endoleak, stent-graft migration, and patent visceral vessels. The femoral access sites were percutaneously repaired using the preclose technique. The postoperative course was uneventful. Whole-exome genetic testing revealed the same missense fibrillin-1 variant mutation 


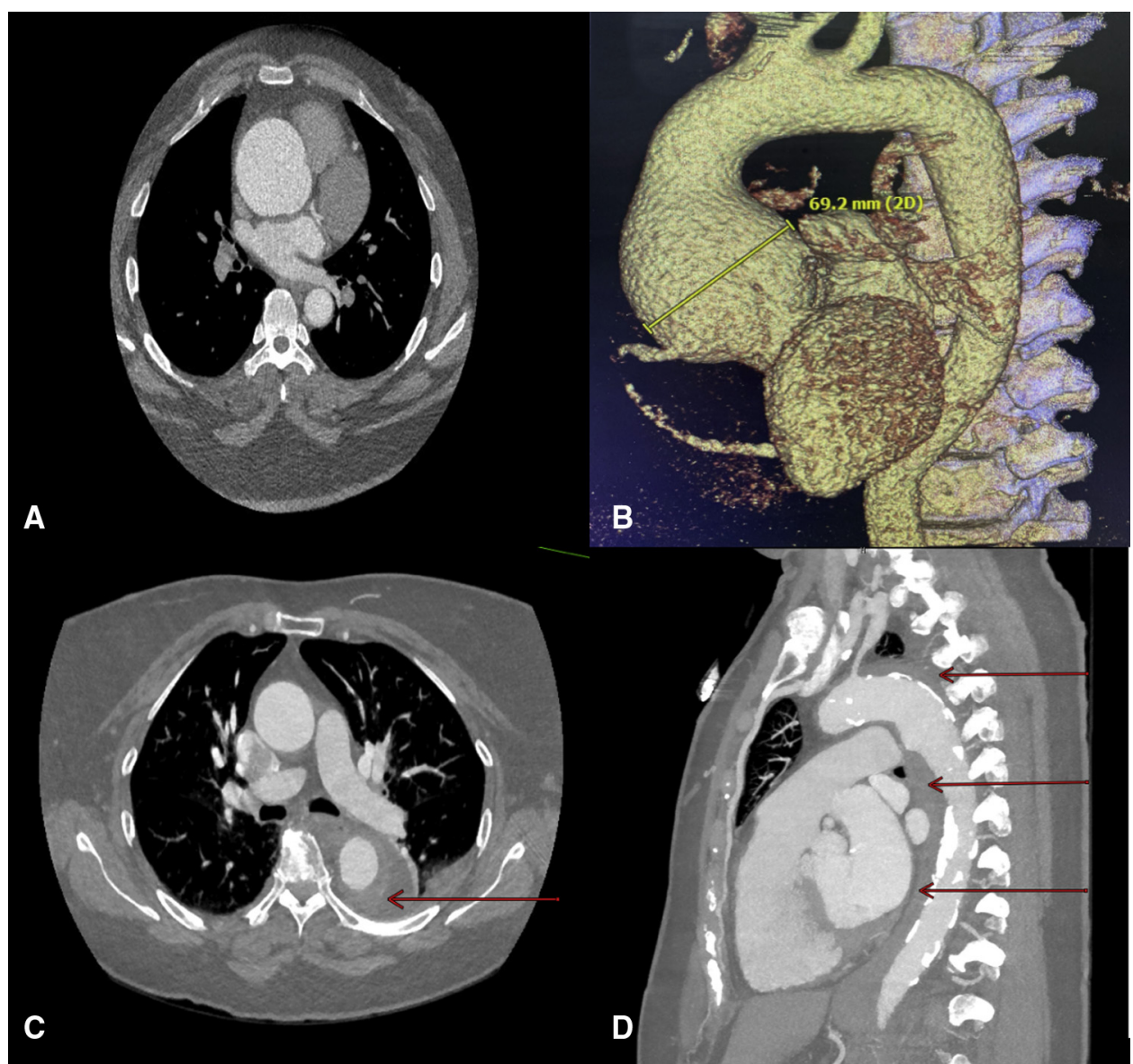

FIGURE 1. Preoperative imaging revealing an aortic root aneurysm and type B intramural hematoma in 2 related family members, respectively, due to a variant fibrillin-1 mutation. A, Computed tomography scan of the chest demonstrating a $6.9-\mathrm{cm} \times 7.3$-cm aortic root aneurysm in a 36-year-old male patient found to have a novel fibrillin-1 mutation; B, 3-Dimensional representation of the aortic root aneurysm in this male patient with a variant fibrillin-1 mutation. C, Computed tomography angiogram depiction of an acute type B intramural hematoma (indicated by red arrow) in a 69-year-old related female patient with the same variant fibrillin-1 mutation. D, Sagittal section of the acute type B intramural hematoma in the related family member (indicated by red arrows).

as in case 1 , and informed consent for publication was obtained from both patients. At 1-month follow-up, she was without chest pain and repeat 6-month CT showed excellent thoracic aortic remodeling with reduction in IMH size $(5 \mathrm{~mm})$.

\section{DISCUSSION}

This report introduces a novel missense variant in the fibrillin-1 gene (c.T556C: p.C186R) and summarizes its phenotypic effects on aortic wall homeostasis, notably an ascending aortic aneurysm and an acute type B aortic IMH and their successful management. Based on American College of Medical Genetics and Genomics guidelines, this rare mutation is classified as likely pathogenic with a population frequency of 0 in 251,000 in the genome aggregation database (gnomAD). ${ }^{1}$ To date, there is only one entry in a Marfan disease-related database, without any phenotypic information for this variant. ${ }^{2}$ This report helps bridge that gap by contributing important phenotypic information for the variant.
Multiple in silico algorithms predict a deleterious effect of this variant on protein function, most likely from cysteine residues (C186). While there are multiple reported germline variants of the fibrillin-1 gene, only a few genotype-phenotype correlations are established., Identification of the culprit variant is essential in planning mode of surgical therapy, genetic counseling, and future surveillance. This is important in the current era of increasing adaptation of endovascular technology for aortic pathologies. While open surgery remains the mainstay of treatment in patients with connective tissue disorders, there are increasing reports on the use of endovascular technology. ${ }^{5-8}$ More studies evaluating newer generations of stent-grafts with long-term data on rates of therapeutic failure, associated reintervention, and effect on aortic remodeling are needed to guide the choice of therapy.

In summary, this report introduces a novel variant mutation in the fibrillin-1 gene (c.T556C: p.C186R) and 
highlights its phenotypic effects of thoracic aortic aneurysms and aortic IMH.

\section{References}

1. Richards S, Aziz N, Bale S, Bick D, Das S, Gastier-Foster J, et al. Standards and guidelines for the interpretation of sequence variants: a joint consensus recommendation of the American College of Medical Genetics and Genomics and the Association for Molecular Pathology. Genet Med. 2015;17:405-24.

2. Groth KA, Von Kodolitsch Y, Kutsche K, Gaustadnes M, Thorsen K, Andersen NH, et al. Evaluating the quality of Marfan genotype-phenotype correlations in existing FBN1 databases. Genet Med. 2017;19:772-7.

3. Aubart M, Gazal S, Arnaud P, Benarroch L, Gross MS, Buratti J, et al. Association of modifiers and other genetic factors explain Marfan syndrome clinical variability. Eur J Hum Genet. 2018;26:1759-72.
4. Frédéric MY, Lalande M, Boileau C, Hamroun D, Claustres M, Béroud C, et al UMD-predictor, a new prediction tool for nucleotide substitution pathogenicity - application to four genes: FBN1, FBN2, TGFBR1, and TGFBR2. Hum Mutat. 2009;30:952-9.

5. Waterman AL, Feezor RJ, Lee WA, Hess PJ, Beaver TM, Martin TD, et al. Endovascular treatment of acute and chronic aortic pathology in patients with Marfan syndrome. J Vasc Surg. 2012;55:1234-40; disucssion 1240-31.

6. Eid-Lidt G, Gaspar J, Melendez-Ramirez G, Cervantes SJ, González-Pacheco H Dámas de Los Santos F, et al. Endovascular treatment of type B dissection in patients with Marfan syndrome: mid-term outcomes and aortic remodeling. Catheter Cardiovasc Interv. 2013;82:E898-905.

7. Kouchoukos NT. Endovascular surgery in Marfan syndrome: CON. Ann Cardiothorac Surg. 2017;6:677-81.

8. Pacini D, Parolari A, Berretta P, Di Bartolomeo R, Alamanni F, Bavaria J. Endovascular treatment for type B dissection in Marfan syndrome: is it worthwhile? Ann Thorac Surg. 2013;95:737-49. 